\title{
Substrate-induced cooperative effects in water adsorption from density functional calculations
}

\author{
Pepa Cabrera-Sanfelix, ${ }^{1}$ M. V. Fernández-Serra, ${ }^{2}$ A. Arnau, ${ }^{1,3,4}$ and D. Sánchez-Portal ${ }^{1,3}$ \\ ${ }^{1}$ Donostia International Physics Center (DIPC), Paseo Manuel de Lardizabal 4, San Sebastian 20018, Spain \\ ${ }^{2}$ Physics and Astronomy, State University of New York, Stony Brook, New York 11794-3800, USA \\ ${ }^{3}$ Centro de Física de Materiales, Materials Physics Center (MPC), CSIC-UPV/EHU, Paseo Manuel de Lardizabal 5, \\ San Sebastián 20018, Spain \\ ${ }^{4}$ Departamento de Física de Materiales, Facultad de Química, UPV/EHU, Apdo. 1072, San Sebastián 20080, Spain
}

(Received 18 June 2010; published 16 September 2010)

\begin{abstract}
Density functional theory calculations are used to investigate the role of substrate-induced cooperative effects on the adsorption of water on a partially oxidized transition-metal surface, $\mathrm{O}(2 \times 2) / \mathrm{Ru}(0001)$. Focusing particularly on the dimer configuration, we analyze the different contributions to its binding energy. A significant reinforcement of the intermolecular hydrogen bond ( $\mathrm{H}$ bond), also supported by the observed frequency shifts of the vibration modes, is attributed to the polarization of the donor molecule when bonded to the $\mathrm{Ru}$ atoms in the substrate. This result is further confirmed by our calculations for a water dimer interacting with a small Ru cluster, which clearly show that the observed effect does not depend critically on fine structural details and/or the presence of coadsorbates. Interestingly, the cooperative reinforcement of the $\mathrm{H}$ bond is suppressed when the acceptor molecule, instead of the donor, is bonded to the surface. This simple observation can be used to rationalize the relative stability of different condensed structures of water on metallic substrates.

DOI: 10.1103/PhysRevB.82.125432

PACS number(s): 33.15.Fm, 68.43. $-\mathrm{h}, 33.15 . \mathrm{Kr}$
\end{abstract}

\section{INTRODUCTION}

The atomic-level understanding of the adsorption of water on metallic substrates and, in particular, on $\mathrm{Ru}(0001)$, has received a lot of attention in recent years. ${ }^{1-12}$ At large water coverage, the determination of the most stable structures of the water adlayer becomes a quite challenging task. This is mainly due to the interplay between two interactions of similar strengths: the intermolecular hydrogen-bond ( $\mathrm{H}$ bond) and the water-metal interaction. ${ }^{3,10}$ In the case of $\mathrm{Ru}$, an additional complication comes from the competing stability of the partially dissociated and the intact-molecule adsorption configurations of water. . $^{50,12}$

Using scanning tunnelling microscopy (STM) supplemented by density functional theory (DFT) calculations, Michaelides and Morgenstern ${ }^{13}$ were recently able to resolve the structures of small water clusters adsorbed on $\mathrm{Cu}(111)$ and $\operatorname{Ag}(111)$. These results help to characterize the ability of water molecules to simultaneously bond to a metallic substrate and to form $\mathrm{H}$ bonds between them and, therefore, to rationalize the observed structures for extended $\mathrm{H}$-bonded water networks on metallic substrates. From a structural point of view, there are at least two types of water molecules: one type forms a direct bond with the substrate, whereas the other is essentially icelike $\mathrm{H}$ bonded and has little interaction with the metal substrate. ${ }^{14}$

From an energetic point of view, the increase in the water coverage on a metallic substrate, up to the complete monolayer, usually enhances the adsorption energy per molecule. This is easily understood since the coordination (and the average number of $\mathrm{H}$ bonds per molecule) increases as extended water networks grow on the substrate. ${ }^{7,10}$ This also occurs on ionic ${ }^{15}$ and graphitic substrates. ${ }^{16}$ However, the formation of extended water networks is typically accompanied by other phenomena that also cause an appreciable increase in the binding energy per water molecule. These are the so-called cooperative effects in the water-water interaction: the strength of the $\mathrm{H}$ bond between two water molecules is largely increased by the fact that those molecules have additional $\mathrm{H}$ bonds with other neighboring molecules. In general, other long-range electrostatic interactions can also favor larger polarization of the water molecules and induce a strengthening of the $\mathrm{H}$ bonds.

The cooperative or nonpairwise character of the interactions is, for example, a fundamental property of liquid water, where $\mathrm{H}$ bonds are up to $250 \%$ stronger than for the isolated $\mathrm{H}$ bond of the dimer. ${ }^{17}$ Cooperative effects are also quite strong in small water clusters. ${ }^{11,18-24}$ The key ingredient to understand these cooperative effects is the polarization induced in each water molecule by the presence of their neighbors. This polarization has its major effect on the lone pairs of the oxygen atoms, which are the electrons involved in the formation of hydrogen bonds. The donor O-H covalent bond also suffers a significant polarization with a net shift of the electron density toward the oxygen of the donor molecule..$^{25}$ These effects lead to the increase in the molecular dipole ${ }^{26}$ and, correspondingly, to the strengthening of the new $\mathrm{H}$ bonds formed with additional water molecules. The quantum character of the protons also contribute to enhance these effects, leading to the elongation of the $\mathrm{O}-\mathrm{H}$ covalent bond in condensed phases and a further increase in the dipole moment. ${ }^{27}$

On forming the $\mathrm{H}$ bond, the donor hydrogen atom moves away from its oxygen and the acceptor lone pair stretches toward the donor hydrogen. Thus, both oxygen atoms are pulled together while the covalent $\mathrm{O}-\mathrm{H}$ bond is being stretched and weakened. ${ }^{28}$ The main origin of this weakening is the Pauli repulsion between the lone pair of the acceptor molecule and the electrons localized on the $\mathrm{O}-\mathrm{H}$ bond of the donor molecule. ${ }^{29}$ Additionally, the formation of the $\mathrm{H}$ bond gives rise to a small (few milielectrons) $)^{1,12,30}$ charge transfer from the lone pair of the acceptor molecule to the donor molecule. This transfer also contributes to the weakening of 
the O-H covalent bond of the donor molecule. ${ }^{31}$ These effects are the reason, for example, for the redshift of the $\mathrm{O}-\mathrm{H}$ stretching frequency in ice versus liquid water. This shift also correlates with a blueshift of the H-bond stretching band in ice as compared to water. So there is a correlation between the strength of these two bonds: the stronger is the $\mathrm{H}$ bond, the weaker the covalent $\mathrm{O}-\mathrm{H}$ bond and the shorter the distance $\mathrm{O} \cdots \mathrm{O}$ between the oxygen atoms of both molecules. Therefore, the weakening and elongation of the O-H covalent bond becomes a good indicator of the stability of the $\mathrm{H}$ bond. ${ }^{32}$ The main observables that can be correlated with the strength of $\mathrm{H}$ bonds are the intermolecular distances and the frequency shifts of the stretching modes of those covalent bonds containing the donor hydrogen atoms.

Much work to date has been devoted to the study of cooperative effects in water networks. However, we can also expect the appearance of substantial cooperative effects in other situations. The necessary condition is that a strong polarization is induced in those water molecules participating in the $\mathrm{H}$ bond and, in particular, in the donor molecule. The adsorption of water on some substrates can provide a mechanism to generate such additional polarization. Indeed, as we will see below, the adsorption of the donor water molecule to some substrates, characterized by a strong oxygen-metal interaction, can give rise to a significant strengthening of the $\mathrm{H}$ bond, comparable to that observed in water and ice.

In the present work, we investigate in detail the intermolecular $\mathrm{H}$ bond in a water dimer interacting with different substrates. Our initial motivation comes from the observation of anomalously large adsorption energies per molecule for a water dimer adsorbed on $\mathrm{O}(2 \times 2) / \mathrm{Ru}(0001) .{ }^{33}$ This structure was initially proposed to explain some of the STM images obtained for water deposited on this substrate at coverages larger than 0.25 ML. Here we perform a quantitative analysis of the different contributions to this large binding energy and conclude that it is mainly due to the strengthened $\mathrm{H}$ bond within the water dimer. The shifts of the calculated vibrations and, in particular, that of $\mathrm{O}-\mathrm{H}$ stretching mode are used to characterize this increase in stability. Afterwards, we use a simple model to explore the effect of adsorption on a general Ru substrate: a water dimer interacting with a small metal cluster. This simple model confirms the validity of our initial conclusions and demonstrates that H-bond stabilization stems primarily from the interaction of the donor molecule with the metallic substrate and does not depend critically on fine structural details or the presence of coadsorbates. Indeed, our work seems to indicate that the appearance of strong cooperative effects in the water-water interaction induced by the adsorption to a substrate is quite general. This seems to be confirmed, for example, by the high binding energy per molecule obtained for a water dimer adsorbed on an ionic substrate such as $\mathrm{NaCl} .{ }^{15,34}$

\section{THEORETICAL METHOD}

Our DFT calculations were performed using the Vienna $a b$ initio simulation package (VASP), ${ }^{35-37}$ within the PerdewWang 1991 (PW91) version of the generalized gradient approximation (GGA). ${ }^{38}$ The projector augmented wave ${ }^{39,40}$ method was used to describe the interaction of valence electrons with the $\mathrm{Ru}, \mathrm{O}$, and $\mathrm{H}$ cores. To describe the $\mathrm{Ru}(0001)$ and $\mathrm{O} / \mathrm{Ru}(0001)$ surfaces we used a symmetric slab containing seven $\mathrm{Ru}$ layers plus a similar amount of vacuum between periodic replicas of the slab. Adsorbates were always placed on both sides of the slab to keep the mirror symmetry and the zero total dipole moment along the normal to the surface, consistent with the periodic boundary conditions. These computational details are similar to those used in our previous work on similar systems. ${ }^{12,33}$ A plane-wave cutoff of $400 \mathrm{eV}$ and a $6 \times 6 \times 1 \mathrm{k}$-point sampling was used for our smallest cell, corresponding to a $2 \times 2$ unit cell in the lateral directions. For the larger $4 \times 4$ unit cell, used to represent lower water coverage, the $k$-point sampling was reduced to $3 \times 3 \times 1$. All geometries were optimized by allowing relaxation of all degrees of freedom of the two outermost $\mathrm{Ru}$ layers and the $\mathrm{O}$ and $\mathrm{H}$ atoms until residual forces were smaller than $0.03 \mathrm{eV} / \AA$. This procedure has been proved to be accurate enough. ${ }^{33}$ The adsorption energies of the water molecules are calculated as described in previous work. ${ }^{33}$ Although it is well known that DFT does not account for dispersion interactions, $\mathrm{H}$ bonds are reasonably well described using the PW91 DFT-GGA functional for exchange and correlation. ${ }^{41}$ For presentation purposes, we have replaced the discrete delta functions by Gaussian functions with a width of $1.5 \mathrm{~cm}^{-1}$ in the plots of vibration density of states (VDOS).

\section{RESULTS AND DISCUSSION}

\section{A. Water dimer on $O(2 \times 2) / \operatorname{Ru}(0001)$}

The saturated first hydration layer on clean $\mathrm{Ru}(0001)$ corresponds to a water coverage $\left(\theta_{w}\right)$ of $2 / 3 \mathrm{ML}$ and follows a commensurate $p(\sqrt{3} \times \sqrt{3}) R 30^{\circ}$ structure ${ }^{42,43}$ whereas $1 / 4$ ML of water is sufficient to saturate the $\mathrm{O}(2 \times 2) / \mathrm{Ru}(0001)$ surface, following a well-ordered $p$ - $(2 \times 2)$ symmetry. ${ }^{33}$ In both cases, the most favorable adsorption site for a water monomer is on $\mathrm{Ru}$ atop sites. In the case of the $\mathrm{O}(2 \times 2)$ / $\mathrm{Ru}(0001)$ surface the hydrogen atoms of the molecule point toward the neighboring preadsorbed $\mathrm{O}$ atoms on the surface to form two long $\mathrm{H}$ bonds. Therefore, at $1 / 4 \mathrm{ML}$ coverage all the preferred adsorption sites in $\mathrm{O}(2 \times 2) / \mathrm{Ru}(0001)$ are occupied.

However, in a recent work $^{33}$ we found that a very stable structure can be obtained for the $\mathrm{O}(2 \times 2) / \mathrm{Ru}(0001)$ surface at a larger coverage of $1 / 2 \mathrm{ML}$. In this case, only half of the water molecules are directly bonded to the metal atoms of the surface while the other half is attached uniquely through $\mathrm{H}$ bonds to the adsorbed water molecules and to the preadsorbed $\mathrm{O}$ atoms on the surface. The resulting structure is based on the water dimer as a building block (see Fig. 1). Surprisingly, this geometry is energetically degenerate with the saturated water overlayer at $1 / 4 \mathrm{ML}$ in which all the molecules occupy preferred adsorption sites and are well attached to the metal. Furthermore, the isolated water dimer is also very stable on this surface, i.e., at much lower water coverage the dimer continues to be a very favorable adsorption configuration. 


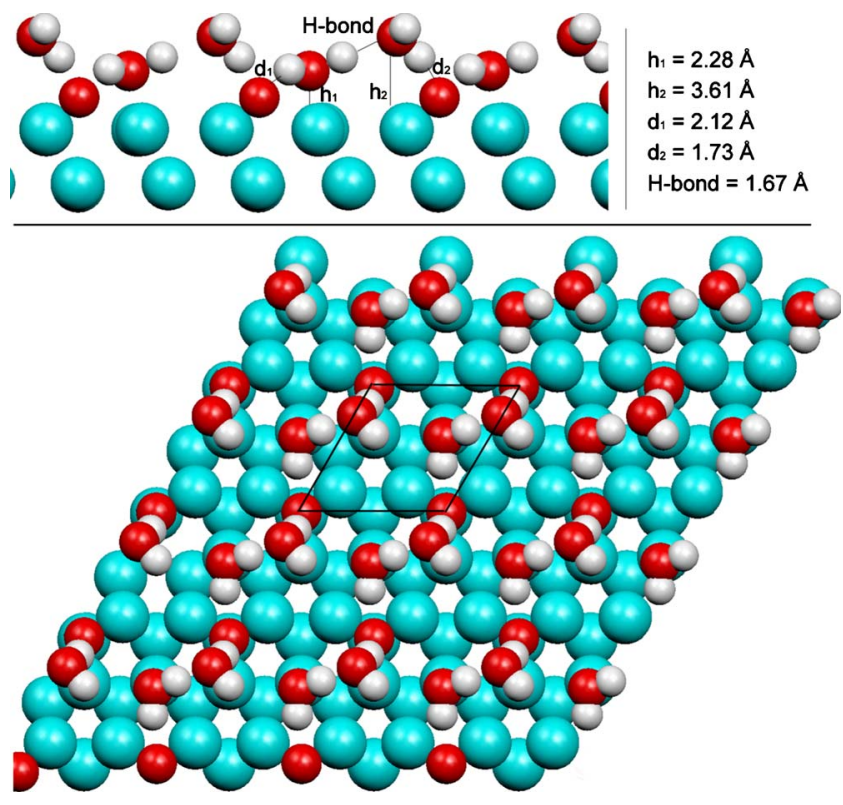

FIG. 1. (Color online) Calculated water configuration at $0.5 \mathrm{ML}$ coverage on the $\mathrm{O}(2 \times 2) / \mathrm{Ru}(0001)$ surface. The structure is formed by dimers in which one of the molecules has its oxygen $2.26 \AA$ above a Ru top site and it is hydrogen bonded to one of the surface oxygen atoms ( $2.12 \AA$ bond length) and to the adjacent water molecule (very short $\mathrm{H}$ bond of $1.67 \AA$ ). The second molecule adsorbs $3.61 \AA$ above the Ru topmost layer and forms a $\mathrm{H}$ bond to the substrate oxygen atom right below $(1.73 \AA$ bond length).

Figure 1 shows these stable water bilayers on $\mathrm{O}(2$ $\times 2) / \mathrm{Ru}(0001)$. The lower laying molecule is well attached to an available $\mathrm{Ru}$ atop site in the cell, its molecular plane slightly tilted and its orientation such that it optimizes a long $\mathrm{H}$ bond with the surface oxygen $(2.12 \AA)$. The second molecule is located $3.61 \AA$ above the surface and forms a $\mathrm{H}$ bond $(1.73 \AA)$ with the surface oxygen atom underneath. The molecules stick together through a relatively short $\mathrm{H}$ bond $(1.67 \AA)$, in which the molecule bonded to the metal acts as the hydrogen donor. The adsorption energy for this relaxed bilayer configuration is $600 \mathrm{meV}$ per water molecule, comparable to the $590 \mathrm{meV}$ found for the saturated layer at $1 / 4$ ML. ${ }^{33}$ At lower coverage the geometry of the water dimer maintains the main characteristics described above. This is the case of the structure described for $\theta_{w} \sim 1 / 8 \mathrm{ML}$ in Ref. 32. This diluted dimer is also energetically degenerate with structures formed by collections of optimally adsorbed monomers at the same coverage. The results for the energetics of the water dimer on $\mathrm{O}(2 \times 2) / \mathrm{Ru}(0001)$ are quite puzzling. On the one hand, according to our analysis, the adsorption energy of the water monomer on $\mathrm{O}(2 \times 2) / \mathrm{Ru}(0001)$ can be approximately divided in two main contributions: (i) the interaction with the metal substrate $\left(E_{\text {metal }}\right)$ that contributes with $\sim 340 \mathrm{meV}$ and (ii) the two long $\mathrm{H}$ bonds $\left(E_{1-\mathrm{Hb}}\right)$ with the surface oxygens, each of them contributing with $\sim 120 \mathrm{meV} .{ }^{33}$ On the other hand, at the adsorbed dimer configuration only one molecule interacts directly with the metal while two $\mathrm{H}$ bonds are formed with the oxygen atoms on the substrate and one $\mathrm{H}$ bond between the two molecules. The
TABLE I. (Color online) Structure and energetics of the freestanding and adsorbed water dimer. Distances in the schemes are given in $\AA$. The binding energies associated with the different hydrogen bond are listed, for the dimer on $\mathrm{O}(2 \times 2) / \mathrm{Ru}(0001)$. We also show the adsorption energy per water molecule.

\begin{tabular}{|c|c|c|c|}
\hline System & Scheme & $\begin{array}{c}\text { H-bond } \\
(\mathrm{meV})\end{array}$ & $\begin{array}{c}E_{\text {ads }} / \mathrm{H}_{2} \mathrm{O} \\
(\mathrm{meV})\end{array}$ \\
\hline $\begin{array}{l}\text { (a) Relaxed water dimer } \\
\text { in vacuum }\end{array}$ & $\Gamma_{105.00}^{0.989} \overbrace{1.981}^{105.3^{\circ}}$ & 237 & --- \\
\hline $\begin{array}{l}\text { (b) Adsorbed water dimer } \\
\text { on } \mathrm{O}(2 \times 2) / \mathrm{Ru}(0001)\end{array}$ & $2.223 \overbrace{}^{106.20^{1.621}} \overbrace{0.003}^{105.8^{\circ}}$ & 724 & 600 \\
\hline
\end{tabular}

calculated $\mathrm{H}$ bond for the free-standing dimer with our method is $\sim 240 \mathrm{meV}$ (see Table I). Thus, a rough estimation of the binding energy per water molecule for the adsorbed dimer structure gives $E_{\mathrm{ads}} \sim\left[E_{\text {metal }}+\left(2 \times E_{1-\mathrm{Hb}}\right)\right.$ $+240] / 2=410 \mathrm{meV} / \mathrm{H}_{2} \mathrm{O}$, which is almost $200 \mathrm{meV} / \mathrm{H}_{2} \mathrm{O}$ smaller than the actual calculated value. Even if the $\mathrm{H}$ bonds formed with the substrate are in average more stable for the dimer than for the monomer (since they are significantly shorter in the former case), the estimated $E_{\text {ads }}$ per molecule would be at least $\sim 100 \mathrm{meV}$ too low. In the following, we study in detail the reasons for this high stability of the adsorbed dimer and conclude that the key ingredient is the strengthening of the intermolecular $\mathrm{H}$ bond due to the chemical interaction of the donor water molecule with the $\mathrm{Ru}$ metal atoms in the substrate.

As a first step, we compare the geometry and energetics of the adsorbed and free-standing water dimer, both computed using a supercell of the same size. The results are presented in Table I. The calculated length of the $\mathrm{H}$ bond for the freestanding dimer (1.89 ̊) is comparable, although slightly shorter, than that obtained with other theoretical methods such as Hartree-Fock (HF/6-311+ $\left.G^{* *}, 1.95 \AA\right)$ or MP2 $\left(\mathrm{MP} 2 / 6-311+G^{* *}, 2.06 \AA\right),{ }^{32,44}$ and the $\mathrm{H}$ bond is $\sim 0.25 \AA$ shorter for the adsorbed dimer. This is an indication of the reinforcement of the intermolecular interaction. In water clusters, the stabilization of the H-bond network has been recognized to increase with the number of molecules forming the cluster. ${ }^{19,45}$ As mentioned in the introduction, the importance of such cooperative effects has been also recognized in liquid water. ${ }^{17}$ In our case, the strengthening of the intermolecular $\mathrm{H}$ bond should come from the interaction with the substrate.

In order to explore this effect, Fig. 2 presents the chargedensity surface $\left(\rho_{\text {dimer }}\right)$ for both, the free-standing dimer and the adsorbed dimer on the $\mathrm{O}(2 \times 2) / \mathrm{Ru}(0001)$ surface. Notice that in the latter case [Fig. 2(b)] the charge density corresponding to the $\mathrm{O}(2 \times 2) / \mathrm{Ru}(0001)$ surface has been subtracted (i.e., $\rho_{\text {dimer }}=\rho_{\text {dimer/surface }}-\rho_{\text {surface }}$ ). The shapes of the density isosurfaces are very similar in both cases. However, the wider section of the isosurface in the intermolecular region for the adsorbed dimer is a clear signature of a larger charge accumulation in that region. This indicates that the interaction with surface indeed influences the intermolecular $\mathrm{H}$ bond. 


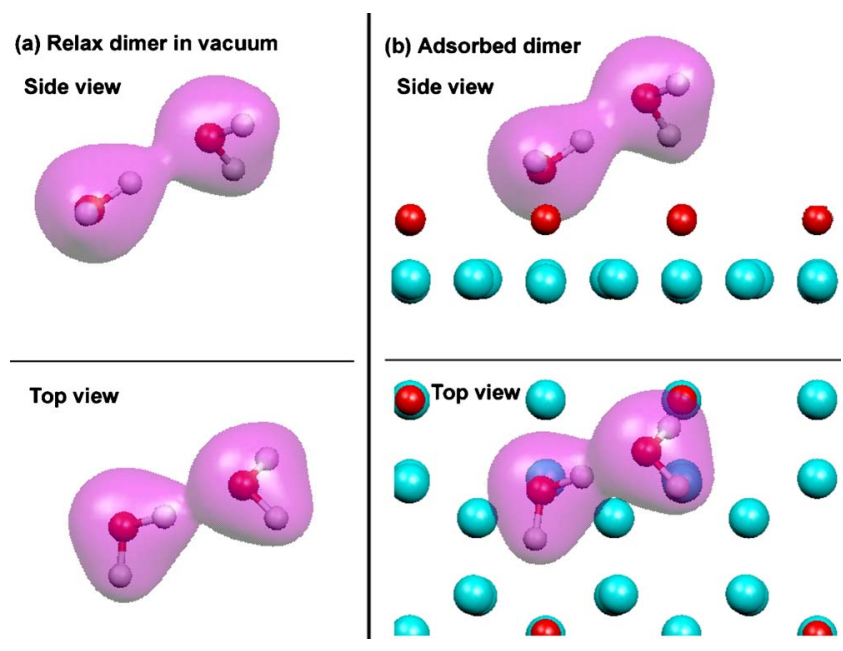

FIG. 2. (Color online) Isosurface of the calculated charge density for a water dimer: (a) free-standing water dimer $\left(\rho_{\text {dimer }}\right)$; (b) adsorbed water dimer on the $\mathrm{O}(2 \times 2) / \mathrm{Ru}(0001)$ surface. Notice that in this second case we have subtracted the charge density corresponding to the surface, i.e., $\rho_{\text {dimer }}=\rho_{\text {dimer/surface }}-\rho_{\text {surface }}$. In both cases, the value of the density used to plot the surfaces is 0.02 a.u.

These effects become more evident when plotting the induced charge density, as shown in Fig. 3. The plot for the free-standing dimer clearly indicates that the formation of the $\mathrm{H}$ bond is accompanied by the polarization of both molecules: electron depletion appears around the $\mathrm{H}$ atom of the donor molecule, whereas charge accumulation, pointing toward the neighboring molecule, is visible on the $\mathrm{O}$ atom of the acceptor molecule. The adsorption of the dimer to the substrate creates a slightly more complex pattern with several contributions [Fig. 3(b)]. In addition to the intermolecular $\mathrm{H}$ bond, the additional bonds formed with the substrate also act as additional sources of polarization. In particular, the interaction of the donor molecule with the $\mathrm{Ru}$ atom underneath gives rise to an appreciable charge rearrangement

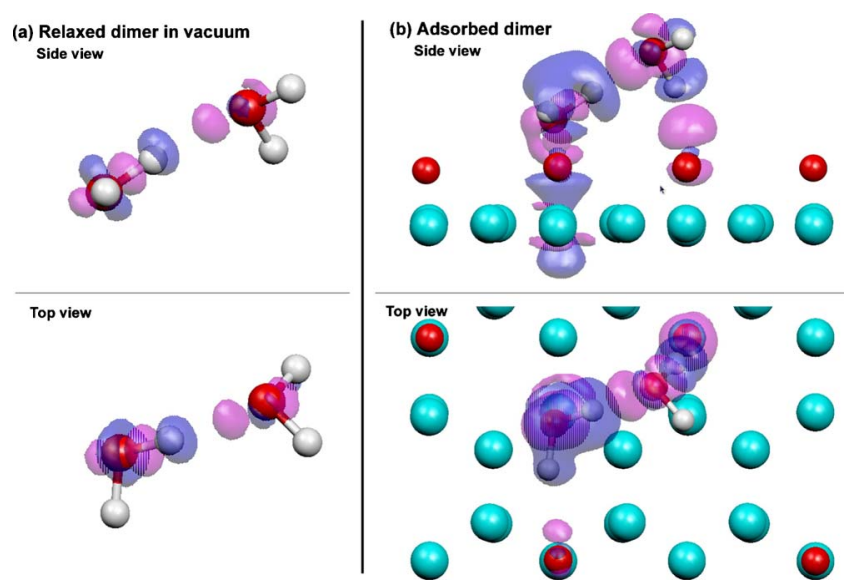

FIG. 3. (Color online) Calculated induced charge density for a water dimer: (a) free-standing dimer $\left(\rho_{\text {ind }}=\rho_{\text {dimer }}-\rho_{\text {monomers }}\right)$; (b) adsorbed water dimer on the $\mathrm{O}(2 \times 2) / \mathrm{Ru}(0001)$ surface $\left(\rho_{\text {ind }}\right.$ $\left.=\rho_{\text {dimer/surface }}-\rho_{\text {surface }}-\rho_{\text {monomers }}\right)$. Light (pink) surfaces correspond to electron accumulations while dark (blue) correspond to electron depletions. Surfaces are plotted for the isovalues \pm 0.003 a.u. characterized by a depletion of electron charge around the $\mathrm{Ru}$ atom and an electron accumulation on the oxygen of the molecule. Therefore, the polarization induced by the interaction of the donor molecule with the metal tends to enhance, rather than to cancel, the polarization pattern induced by the intermolecular $\mathrm{H}$ bond. It is interesting to notice that similar charge density rearrangements have been recently presented by Michaelides and Morgenstern for the adsorption of water hexamers on $\mathrm{Cu}(111) \cdot{ }^{13}$ In these buckled clusters only half of the molecules are well attached to the $\mathrm{Cu}$ atoms and, therefore, are reminiscent of the dimer structures considered here. According to Michaelides and Morgenstern, the substrateinduced polarization of these water molecules in the buckled-hexamer increases the adsorption energy per water molecule by more than $100 \mathrm{meV}$ with respect to that of a forced planar hexamer. Therefore, the results contained in Fig. 3 are indicative of an important effect of the substrate in the polarization of the molecules and H-bond enhancement. We have, however, performed a more quantitative estimation of the influence of the substrate on the intermolecular $\mathrm{H}$ bond, which is presented in what follows.

Table II presents the different contributions to the energetics of the water dimer adsorbed on the $\mathrm{O}(2 \times 2) / \mathrm{Ru}(0001)$ substrate. The intermolecular interaction in the isolated dimer keeping the geometry of the adsorbed configuration (i) is $\sim 131 \mathrm{meV}$. The dimer-substrate interaction, (ii) $\sim 1.15 \mathrm{eV}$, is estimated by subtracting the energies of the isolated dimer and of the $\mathrm{O}(2 \times 2) / \mathrm{Ru}(0001)$ surface, again both keeping the geometry of the combined relaxed system, from the total energy of the optimized adsorbed water dimer on $\mathrm{O}(2 \times 2) / \mathrm{Ru}(0001)$. Two additional single-point totalenergy calculations have been performed taking out each water molecule from the optimized adsorbed configuration [schemes (iii) and (iv) in Table II], while keeping the geometry of the rest of the system. The corresponding energy values represent the interaction of each of those water molecules with both, the substrate and the remaining molecule. Then, the strength of the intermolecular $\mathrm{H}$ bond can be determined by adding both energies (iii) and (iv) and subtracting the dimer-substrate interaction (ii). With this procedure, the intermolecular $\mathrm{H}$ bond has been estimated to contribute $\sim 724 \mathrm{meV}$ to the stabilization of the system. This value is much higher than the $\sim 131 \mathrm{meV}$ for the $\mathrm{H}$ bond of the fixed-geometry dimer in vacuum or the $237 \mathrm{meV}$ binding energy of the optimized free-standing dimer. Thus, the substrate-induced polarization of the donor molecule makes the intermolecular $\mathrm{H}$ bond of the adsorbed water dimer at least three times stronger than for the free-standing dimer in vacuum.

The polarization of the water molecule, that strengthens the intermolecular $\mathrm{H}$ bond, is also expected to cause a simultaneous weakening of the covalent $\mathrm{O}-\mathrm{H}$ bonds within the molecule. Figure 4 shows the VDOS of both, the relaxed free-standing and the adsorbed water dimer on the $\mathrm{O}(2$ $\times 2) / R u(0001)$ surface. The calculated energy range of the stretching of $\mathrm{O}-\mathrm{H}$ bonds for the free-standing dimer $\left(3500-3800 \mathrm{~cm}^{-1}\right)$ is in good agreement with that found in the experiments. ${ }^{21,46}$ As expected, the stretching mode of the donating O-H covalent bond (between atoms 2 and 3 in Fig. 4) has the lowest frequency, reflecting the weakening of this 
TABLE II. (Color online) Analysis of the different contributions to the binding energy of the adsorbed water dimer on $\mathrm{O}(2$ $\times 2) / \operatorname{Ru}(0001)$.

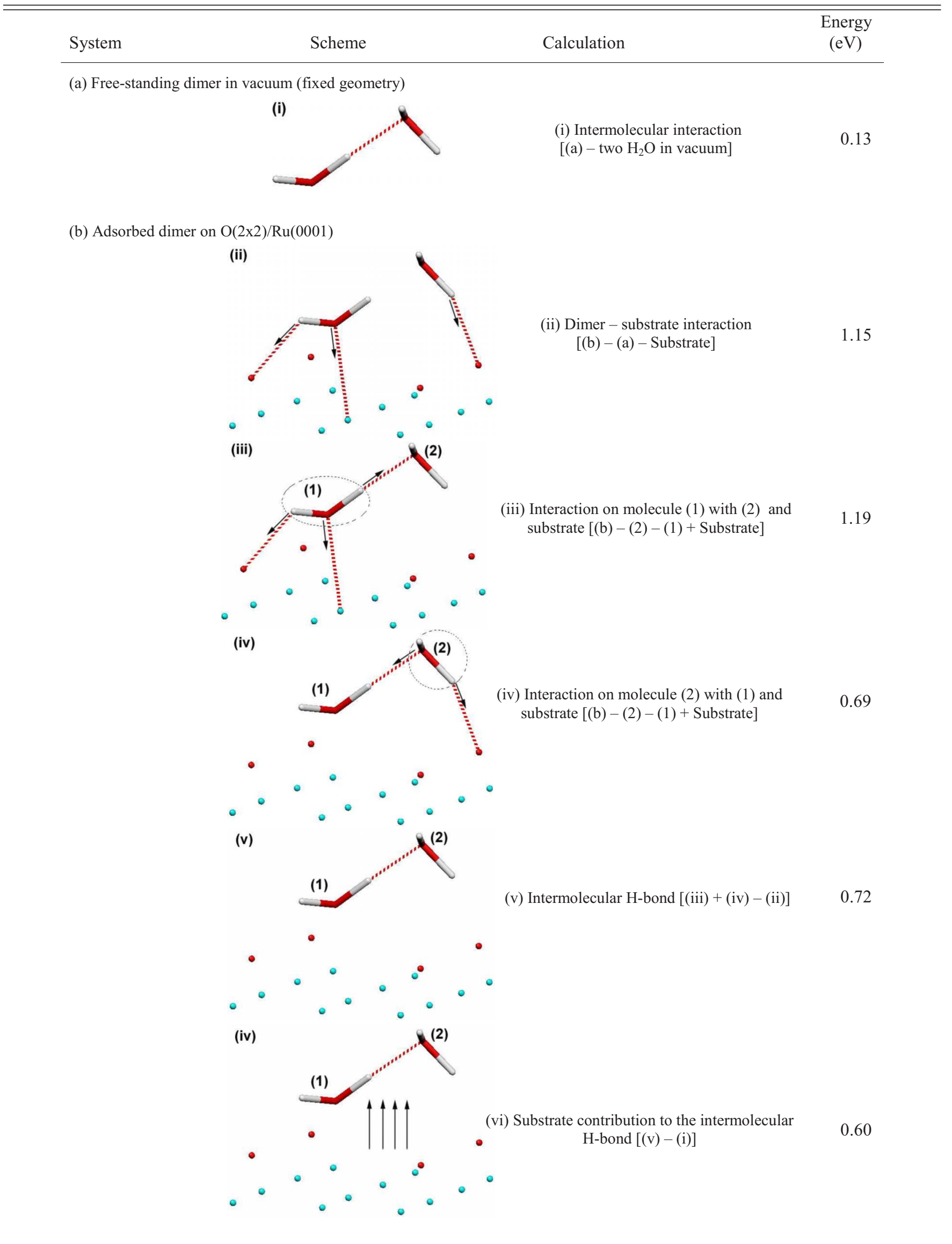


$\mathrm{O}-\mathrm{H}$ bond. For the adsorbed dimer the stretching modes cover a larger frequency range $\left(2950-3800 \mathrm{~cm}^{-1}\right)$. In particular, as a clear signature of the strong intermolecular $\mathrm{H}$ bond, the frequency of the stretching mode of the donating $\mathrm{O}-\mathrm{H}$ bond in this case shifts downward more than $700 \mathrm{~cm}^{-1}$ with respect to the stretching of those $\mathrm{O}-\mathrm{H}$ bonds not involved in $\mathrm{H}$ bonds. This finding is in agreement with the experimental results by Morgenstern and Nieminen, ${ }^{11}$ that observed using inelastic electron tunnelling spectroscopy a large redshift of $\sim 750 \mathrm{~cm}^{-1}$, compared to gas phase, of the $\mathrm{OH}$ stretching for extended structures of ice adsorbed on $\mathrm{Ag}(111)$. As pointed out by these authors this, redshift is too large to be only attributed to the increase in the coordination number of the water molecules in the adsorbed water layer. However, in the light of our calculations, we can interpret this measurement as an evidence of the important role of water-metal bond on the reinforcement of the intermolecular $\mathrm{H}$ bond. The stretching modes of $\mathrm{O}-\mathrm{H}$ bonds involved in the formation of other $\mathrm{H}$ bonds with surface oxygens are also shifted down. For example, the stretching of the O-H bond between atoms 5 and 6 [see Fig. 4(b)] decreases by $\sim 450 \mathrm{~cm}^{-1}$ with respect to the free-standing case. This shift is even larger than the associated with the formation of the intermolecular $\mathrm{H}$ bond in the free-standing dimer and is a signature of an important strengthening of the $\mathrm{H}$ bond with the oxygen atoms on the substrate. This shows that, in addition to the binding with the metal, the fact that the molecule forms more than one $\mathrm{H}$ bond also tends to increase the stability of the whole $\mathrm{H}$-bonded network as already observed for free-standing water clusters. ${ }^{21,24}$ An interesting point to consider is that the weakening of the covalent $\mathrm{O}-\mathrm{H}$ bonds for the adsorbed water dimer and other buckled structures on the oxidized $\mathrm{Ru}$ substrate, associated with the formation of strong $\mathrm{H}$ bonds, may have consequences for the chemistry of water. For example, a reduction in the energy barriers for partial dissociation can be expected in some of these struc-
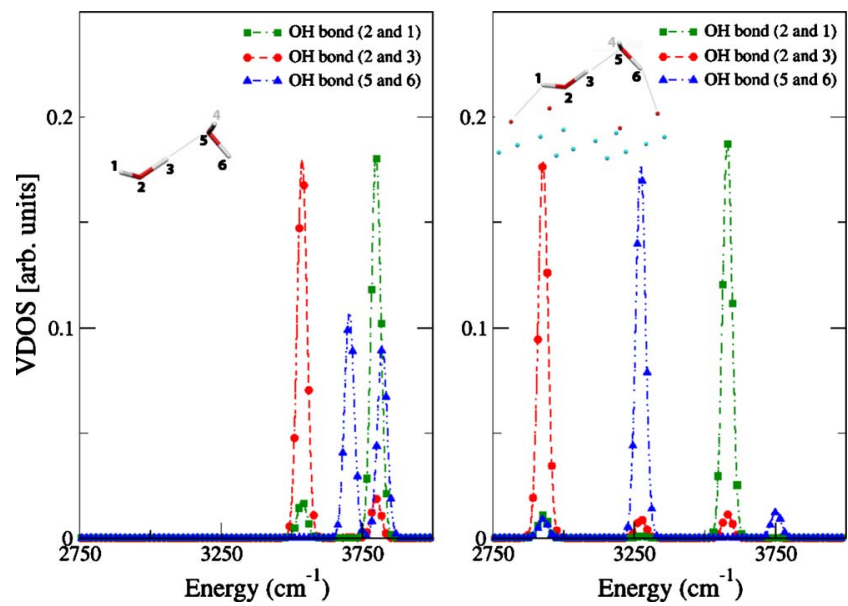

FIG. 4. (Color online) VDOS of the relaxed free-standing water dimer (left panel) and of the adsorbed dimer on the $\mathrm{O}(2$ $\times 2) / \mathrm{Ru}(0001)$ surface (right panel). Only the contributions to the VDOS from those $\mathrm{H}$ atoms involved in the $\mathrm{H}$ bonds of the adsorbed dimer have been included in both panels. tures. The experimental observation that the presence of preadsorbed oxygen, at low coverage, on $\mathrm{Ru}(0001)$ promotes the dissociation of water can support this interpretation. ${ }^{2,6}$

\section{B. Role of the water-metal interaction}

Finally, we perform a meticulous analysis of the watermetal interaction on the observed reinforcement of the $\mathrm{H}$ bond. In order to do this, we investigate the effect of approaching a small metal cluster, a Ru tetrahedron, to a water dimer in vacuum. At each step the metal atoms and the oxygen atom of the donor molecule remain fixed while the rest of the atoms are allowed to relax. The distance $\left(d_{\mathrm{Ru}-\mathrm{O}}\right)$ between the apex of the Ru cluster and the oxygen of the donor molecule varied from $3.72 \AA$ to $2.25 \AA$, the last value corresponding to the optimum $\mathrm{Ru}-\mathrm{O}$ distance. The resulting geometries can be found in Figs. 5(a)-5(d). The $\mathrm{H}$ bond between the water molecules becomes shorter (up to a $\sim 9.5 \%$ shorter) as the donor water molecule approaches the Ru cluster, being a clear signature of the $\mathrm{H}$-bond reinforcement by the metallic cluster. This is confirmed by our energetic calculations illustrated in Fig. 5(e). The interaction energy for each configuration $E_{A-B}^{i n t}$ is calculated by comparing the energies from single-point calculations of fragments $\mathrm{A}$ and $\mathrm{B}$ defined in Fig. 5(a) with that of the combined system. Fragment A comprises the cluster plus the donor water molecule while fragment $\mathrm{B}$ is the acceptor water molecule. The changes on $\Delta E_{A-B}^{i n t}$ as the dimer approaches the Ru cluster can be used to estimate the change in the H-bond strength, i.e., $\Delta E_{A-B}^{i n t} \approx \Delta E_{H \text {-bond. }} \Delta E_{A-B}^{\text {int }}$ increases up to $\sim 180 \mathrm{meV}$ as a result of the interaction of the donor molecule with the metal cluster. Comparing this variation in $\Delta E_{A-B}^{i n t}$ with the $\mathrm{H}$ bond in a free-standing dimer (see Table I), we conclude that solely the interaction of the donor water molecule with the metal can already account for an increase of at least a $75 \%$ in the strength of the intermolecular $\mathrm{H}$ bond.

Figures 6(a)-6(d) show the induced charge-density surface as we approach the water dimer to the Ru tetrahedron. The strong polarization of the system as $d_{\mathrm{Ru}-\mathrm{O}}$ decreases gives a good insight of the reinforcement of the dimer hydrogen bond. As the dimer comes closer to the Ru cluster the metal strongly polarizes the donor molecule. A growing electron depletion appears in the $\mathrm{Ru}$ atoms closer to the donor molecule, similar to what we already observed for the dimer on $\mathrm{O}(2 \times 2) / \mathrm{Ru}(0001)$ substrate [see Fig. 3(b)]. Correspondingly, we observe the increasing polarization, and consequent weakening, of the donating $\mathrm{O}-\mathrm{H}$ covalent bond. In turn, the polarization of the donor molecule also induces an additional polarization in the acceptor, as can be seen in the increasing electron accumulation surrounding the oxygen atom of that molecule. This combined effect reinforces the $\mathrm{H}$ bond as the metal is approached.

We have checked that the large stabilization of the dimer is indeed related to the polarization of the donor molecule and the corresponding $\mathrm{H}$-bond strengthening. If instead of the donor we approach the acceptor molecule to the metal cluster, the effect is the opposite and the dimer is destabilized 

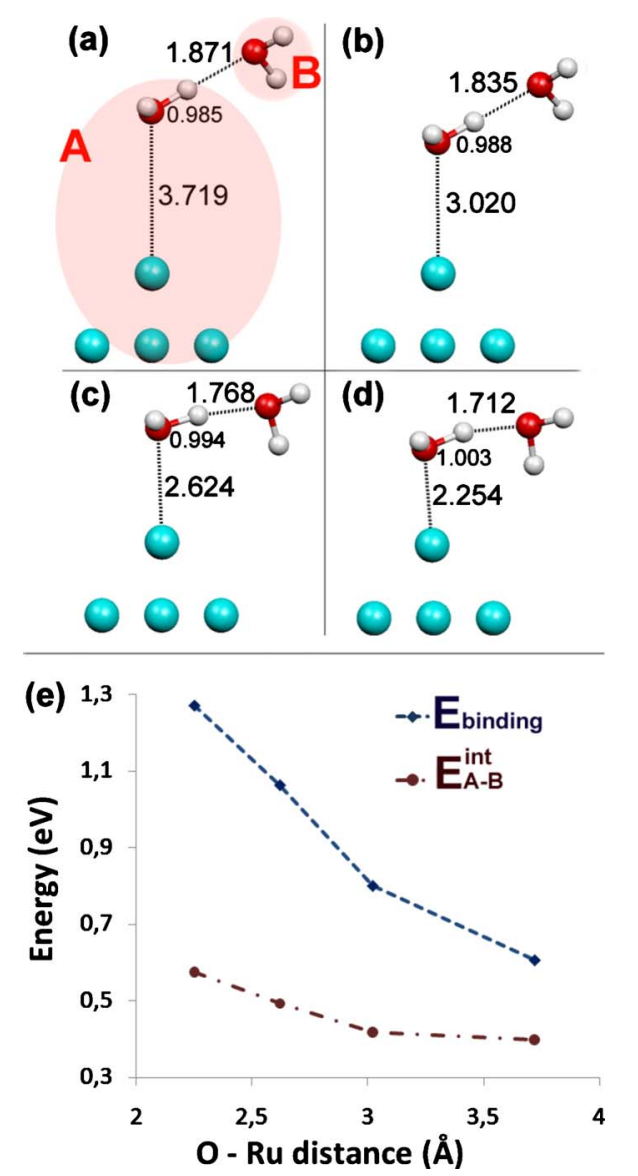

FIG. 5. (Color online) [(a)-(d)] Relaxed structures of a water dimer interacting with a small Ru cluster. The distance between the cluster apex and the oxygen atom of the donor molecule is kept constant during the relaxation. Case (d) corresponds to the optimum $\mathrm{Ru}-\mathrm{O}$ separation. All distances are given in $\AA$. Panel (e) shows the energetics of the system. $E_{A-B}^{i n t}$ is the interaction energy of the fragments A (cluster+donor molecule) and B (acceptor molecule) defined in panel (a). The changes in $E_{A-B}^{i n t}\left(\Delta E_{A-B}^{i n t}\right)$ give a direct estimation of the $\mathrm{H}$-bond strength variation with the $\mathrm{Ru}-\mathrm{O}$ distance. $E_{\text {binding }}$ represents the total binding energy of the system respect to their components, i.e., the isolated cluster and two isolated water molecules.

[see Fig. 6(e)]. For the optimum distance between the acceptor molecule and the cluster apex, the $\mathrm{H}$ bond elongates to $2.192 \AA(\sim 17 \%)$ and the total binding energy of the system is reduced by $\sim 350 \mathrm{meV}$ compared with the optimum binding between the $\mathrm{Ru}$ cluster to the donor molecule. In this case, although the interaction with the metal also polarizes the acceptor molecule, the presence of the neighboring metal efficiently screens the charge of the oxygen atom in the acceptor molecule and, therefore, the $\mathrm{H}$ bond is strongly weakened. Thus, these calculations clarify the role of the substrate on the adsorption of water: strong substrate-induced cooperative effects in the H-bond network of water are directly associated with the preferential adsorption of the donor molecules to the substrate. This is in agreement with previous work on small water clusters adsorbed on metallic substrates, such trimers on Pt (Ref. 47) and hexamers on $\mathrm{Cu}{ }^{13}$

In summary, from the calculations presented in this section we can conclude that, quite independently of the struc-

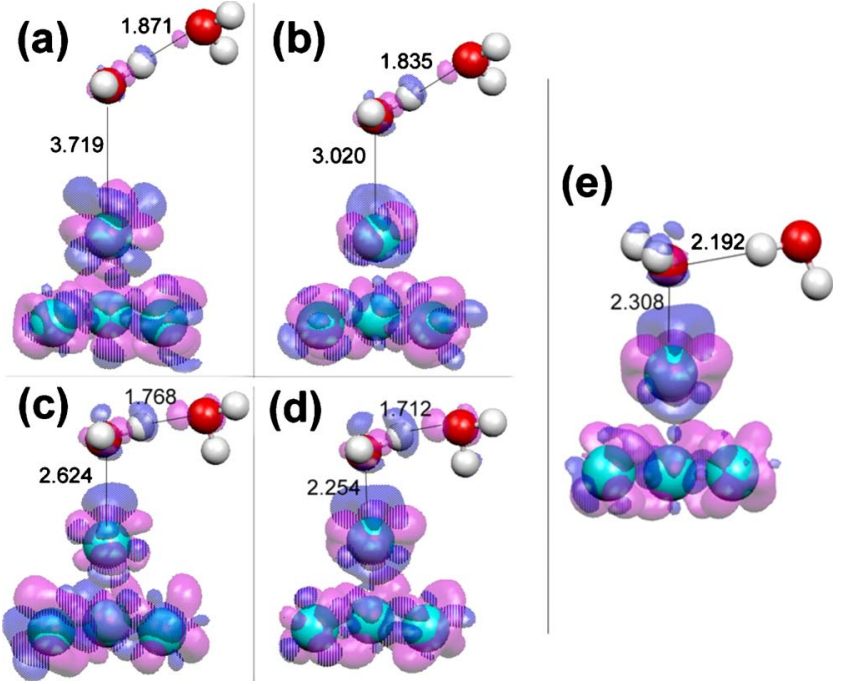

FIG. 6. (Color online) Panels (a)-(d): calculated induced charge density for the systems presented in Fig. 5. The induced charge is calculated as $\rho_{\text {induced }}=\rho_{\text {dimer/cluster }}-\rho_{\text {donor }}-\rho_{\text {acceptor }}-\rho_{\text {cluster }}$, where $\rho_{\text {dimer/cluster }}$ is the electron density of the combined system, $\rho_{\text {donor }}$ and $\rho_{\text {acceptor }}$ are the densities of the isolated water molecules, and $\rho_{\text {cluster }}$ that of the isolated $\mathrm{Ru}$ cluster. Light (pink) surface correspond to the isovalue +0.004 a.u. (electron accumulation) and the dark (blue) surface correspond to isovalue -0.004 a.u. (electron depletion). Panel (e) shows the relaxed configuration when the dimer is interacting with the cluster through the acceptor molecule, along with the corresponding induced charge $\rho_{\text {induced }}$. All distances are given in $\AA$.

tural details of the surface, the interaction with a Ru substrate can give rise to a significant stabilization of the $\mathrm{H}$ bonds between water molecules when the bonding to the substrate takes place through the donor molecule. Presumably this is also the case for other metallic substrates, as the calculations in Refs. 13 and 44, respectively, for $\mathrm{Pt}$ and $\mathrm{Cu}$, indicate.

\section{CONCLUSIONS}

Motivated by the large stability of a water dimer on $\mathrm{O}(2 \times 2) / \mathrm{Ru}(0001)$ as recently calculated by some of us, ${ }^{33}$ we have studied the role of substrate-induced cooperative effects in the adsorption of water. We have performed DFT calculations for two sets of systems: a water dimer on $\mathrm{O}(2$ $\times 2) / \mathrm{Ru}(0001)$ and a water dimer interacting with a small $\mathrm{Ru}$ cluster. A significant strengthening of the intermolecular $\mathrm{H}$ bond, accompanied by the corresponding weakening of the donating O-H covalent bond, is observed for the studied systems. The reinforcement of the $\mathrm{H}$ bond is due to the strong polarization induced by the interaction with the metallic substrate in the donor molecule. We thus confirm the importance of cooperative effects on the water adsorption on metallic substrates, in agreement with the recent findings ${ }^{11,13,44}$ for water adsorption on $\mathrm{Ag}, \mathrm{Cu}$, and Pt substrates.

Two important consequences can be extracted from our results. (i) It is particularly interesting to note that the $\mathrm{H}$-bond reinforcement only takes place when the water donor molecules are the ones directly bonded to the substrate. This 
provides a simple route to propose sensible structural candidates for water bilayers on metallic substrates. Therefore, in general, those structures in which the binding to the metal takes place through the donor molecule can be expected to be significantly more stable than those in which the acceptor water molecules are attached to the substrate. For example, this provides a very simple rationalization of the results found in Ref. 45.

(ii) Our results for the $\mathrm{O}(2 \times 2) / \mathrm{Ru}(0001)$ surface show that, even in the presence of coadsorbates such as oxygen atoms, the metal-water interaction is the main source of substrate-induced polarization of the water molecules, and thus of substrate-induced cooperative effects. This is quite interesting since the oxygen atoms on the surface can cause an important polarization of the substrate and are able to form $\mathrm{H}$ bonds with the adsorbed water molecules. Still the water-metal interaction seems to have a key role in the appearance of cooperative effects.

In the present work, we have analyzed in detail the role of substrate-induced cooperative effects on the adsorption of water on clean and decorated metallic substrates. Such cooperative effects will be present for all cases in which the water-substrate interaction can cause a significant polarization of the molecule. Surfaces of ionic crystals represent good substrate examples where such effects could be expected. Indeed, the high stability of the structures based on the dimer motif on $\mathrm{NaCl}(001)$, as compared to those based on well-adsorbed monomers, can be interpreted as a manifestation of cooperative effects coming into play. ${ }^{15,34}$ However, in those cases the energy difference between both configurations was much smaller than that found in the present work for adsorption on $\mathrm{Ru}$ surfaces.
We can now speculate with the implications of our findings for the chemistry of water. Since the reinforcement of the $\mathrm{H}$ bond and polarization of the molecule typically takes place at the expense of weakening the donating covalent $\mathrm{O}-\mathrm{H}$ bond, we can expect a decrease in the energy barrier for partial dissociation for some of the structures studied in this paper and, in general, for the water bilayers and other buckled H-bonded networks on metallic substrates. In particular, the behavior found for the $\mathrm{O}(2 \times 2) / \mathrm{Ru}(0001)$ seems to agree with the experimental observation that coadsorption of small amount of oxygen with water on $\mathrm{Ru}(0001)$ enhance the dissociation of water. ${ }^{2,6}$ Besides the effect of the adsorbed oxygen on the energetics of the dissociation products studied in Ref. 12, one could argue that the presence of oxygen is likely to favor the presence of buckled structures similar to the water dimer studied here and reminiscent of the bilayer. These structures would favor the appearance of important cooperative effects and the reduction in the dissociation barriers. However, further work is necessary to calibrate the actual importance of substrate-induced cooperative effects on the partial dissociation of water on metallic substrates.

\section{ACKNOWLEDGMENTS}

We acknowledge support from Basque Departamento de Educación, UPV/EHU (Grant No. IT-366-07), the Spanish Ministerio Innovación y Ciencia (Grant No. FIS2007-66711C02-00), and the ETORTEK research program funded by the Basque Departamento de Industria and the Diputación Foral de Guipúzcoa. M.V.F.-S. acknowledges support from DOE under Grant No. DE-FG02-09ER16052.
${ }^{1}$ K. Andersson, A. Nikitin, L. G. M. Pettersson, A. Nilsson, and H. Ogasawara, Phys. Rev. Lett. 93, 196101 (2004).

${ }^{2}$ C. Clay, S. Haq, and A. Hodgson, Chem. Phys. Lett. 388, 89 (2004).

${ }^{3}$ D. N. Denzler, C. Hess, R. Dudek, S. Wagner, Ch. Frischkorn, M. Wolf, and G. Ertl, Chem. Phys. Lett. 376, 618 (2003).

${ }^{4}$ N. S. Faradzhev, K. L. Kostov, P. Feulner, T. E. Madey, and D. Menzel, Chem. Phys. Lett. 415, 165 (2005).

${ }^{5}$ P. J. Feibelman, Sci. 295, 99 (2002).

${ }^{6}$ M. J. Gladys, A. A. El-Zein, A. Mikkelsen, J. N. Anderson, and G. Held, Phys. Rev. B 78, 035409 (2008).

${ }^{7}$ S. Haq, C. Clay, G. R. Darling, G. Zimbitas, and A. Hodgson, Phys. Rev. B 73, 115414 (2006).

${ }^{8}$ M. A. Henderson, Surf. Sci. Rep. 46, 5 (2002).

${ }^{9}$ S. Meng, E. G. Wang, and S. W. Gao, Phys. Rev. B 69, 195404 (2004).

${ }^{10}$ A. Michaelides, A. Alavi, and D. A. King, J. Am. Chem. Soc. 125, 2746 (2003).

${ }^{11}$ K. Morgenstern and J. Nieminen, Phys. Rev. Lett. 88, 066102 (2002).

${ }^{12}$ P. Cabrera-Sanfelix, A. Arnau, A. Mugarza, T. K. Shimizu, M. Salmeron, and D. Sánchez-Portal, Phys. Rev. B 78, 155438 (2008).
${ }^{13}$ A. Michaelides and K. Morgenstern, Nature Mater. 6, 597 (2007).

${ }^{14}$ A. Shavorskiy, M. J. Gladys, and G. Held, Phys. Chem. Chem. Phys. 10, 6150 (2008).

${ }^{15}$ P. Cabrera-Sanfelix, A. Arnau, G. R. Darling, and D. SanchezPortal, J. Chem. Phys. 126, 214707 (2007).

${ }^{16}$ P. C. Sanfelix, S. Holloway, K. W. Kolasinski, and G. R. Darling, Surf. Sci. 532-535, 166 (2003).

${ }^{17}$ W. A. P. Luck, J. Mol. Struct. 448, 131 (1998).

${ }^{18}$ L. Gonzalez, O. Mo, M. Yanez, and J. Elguero, J. Mol. Struct.: THEOCHEM 371, 1 (1996).

${ }^{19}$ F. N. Keutsch and R. J. Saykally, Proc. Natl. Acad. Sci. U.S.A. 98, 10533 (2001).

${ }^{20}$ R. Ludwig and A. Appelhagen, Angew. Chem., Int. Ed. 44, 811 (2005).

${ }^{21}$ K. Ohno, M. Okimura, N. Akai, and Y. Katsumoto, Phys. Chem. Chem. Phys. 7, 3005 (2005).

${ }^{22}$ V. S. Znamenskiy and M. E. Green, J. Chem. Theory Comput. 3, 103 (2007).

${ }^{23}$ O. A. Loboda and V. V. Goncharuk, Journal of Water Chemistry and Technology 31, 98 (2009).

${ }^{24}$ A. M. Tokmachev, A. L. Tchougreeff, and R. Dronskowski, ChemPhysChem 11, 384 (2010). 
${ }^{25}$ In the nomenclature used throughout the paper, donor molecule refers to the water molecule whose hydrogen atom is pointing to and interacts with the electronegative oxygen atom of the acceptor water molecule.

${ }^{26}$ P. L. Silvestrelli and M. Parrinello, J. Chem. Phys. 111, 3572 (1999).

${ }^{27}$ B. Chen, I. Ivanov, M. L. Klein, and M. Parrinello, Phys. Rev. Lett. 91, 215503 (2003).

${ }^{28}$ C. Kozmutza, I. Varga, and L. Udvardi, J. Mol. Struct.: THEOCHEM 666-667, 95 (2003).

${ }^{29}$ M. V. Fernández-Serra and E. Artacho, Phys. Rev. Lett. 96, 016404 (2006).

${ }^{30}$ R. Z. Khaliullin, A. T. Bell, and M. Head-Gordon, Chem.-Eur. J. 15, 851 (2009).

${ }^{31}$ J. E. Bertie, H. J. Labbe, and E. Whally, J. Chem. Phys. 50, 4501 (1969).

${ }^{32}$ S. J. Grabowski, Chem. Phys. Lett. 338, 361 (2001).

${ }^{33}$ P. Cabrera-Sanfelix, D. Sanchez-Portal, A. Mugarza, T. K. Shimizu, M. Salmeron, and A. Arnau, Phys. Rev. B 76, 205438 (2007).
${ }^{34}$ Y. Yang, S. Meng, and E. G. Wang, Phys. Rev. B 74, 245409 (2006).

${ }^{35}$ G. Kresse and J. Hafner, Phys. Rev. B 47, 558 (1993).

${ }^{36}$ G. Kresse and J. Hafner, Phys. Rev. B 49, 14251 (1994).

${ }^{37}$ G. Kresse and J. Furthmuller, Phys. Rev. B 54, 11169 (1996).

${ }^{38}$ J. P. Perdew, J. A. Chevary, S. H. Vosko, K. A. Jackson, M. R. Pederson, D. J. Singh, and C. Fiolhais, Phys. Rev. B 46, 6671 (1992).

${ }^{39}$ P. E. Blöchl, Phys. Rev. B 50, 17953 (1994).

${ }^{40}$ G. Kresse and D. Joubert, Phys. Rev. B 59, 1758 (1999).

${ }^{41}$ K. Laasonen, F. Csajka, and M. Parrinello, Chem. Phys. Lett. 194, 172 (1992).

${ }^{42}$ G. Held and D. Menzel, Surf. Sci. 316, 92 (1994).

${ }^{43}$ M. M. Thiam, T. Kondo, N. Horimoto, H. S. Kato, and M. Kawai, J. Phys. Chem. B 109, 16024 (2005).

${ }^{44}$ S. J. Grabowski, J. Phys. Chem. A 105, 10739 (2001).

${ }^{45}$ R. Ludwig, Phys. Chem. Chem. Phys. 4, 5481 (2002).

${ }^{46}$ D. A. Schmidt and K. Miki, ChemPhysChem 9, 1914 (2008).

${ }^{47}$ T. Lankau, K. Nagorny, and I. L. Cooper, Langmuir 15, 7308 (1999). 